\title{
Dynamics of a two-phase flow through a minichannel: Transition from churn to slug flow
}

\author{
Grzegorz Górski ${ }^{1}$, Grzegorz Litak ${ }^{2,3, a}$, Romuald Mosdorf ${ }^{1}$, and Andrzej Rysak ${ }^{2}$ \\ ${ }^{1}$ Faculty of Mechanical Engineering, Bialystok University of Technology, Wiejska 45C, PL-15-351 Bialystok, Poland \\ ${ }^{2}$ Faculty of Mechanical Engineering, Lublin University of Technology, Nadbystrzycka 36, PL-20-618 Lublin, Poland \\ 3 Department of Process Control, AGH University of Science and Technology, Mickiewicza 30, PL-30-059 Krakow, Poland
}

Received: 28 January 2016 / Revised: 8 March 2016

Published online: 21 April 2016

(C) The Author(s) 2016. This article is published with open access at Springerlink.com

\begin{abstract}
The churn-to-slug flow bifurcations of two-phase (air-water) flow patterns in a 2 mm diameter minichannel were investigated. With increasing a water flow rate, we observed the transition of slugs to bubbles of different sizes. The process was recorded by a digital camera. The sequences of light transmission time series were recorded by a laser-phototransistor sensor, and then analyzed using the recurrence plots and recurrence quantification analysis (RQA). Due to volume dependence of bubbles velocities, we observed the formation of periodic modulations in the laser signal.
\end{abstract}

\section{Introduction}

Two-phase flow in a minichannel is a complex phenomenon which depends on the physical properties of given phases as: density, viscosity, surface tension as well as the channel geometry. In the minichannel one can observe various flows types including isolated, confined, and elongated bubbles, slugs and semi-annular flows, as well as churn and annular flows [1-3]. The liquid flows which accompany the bubble flows can be laminar and turbulent depending on the liquid and gas flow rates [4]. The distance between bubbles or slugs in a minichannel can vary in time. Depending on the flow conditions subsequent distances between bubbles or slugs can change in a periodic or non-periodic way. A high liquid velocity causes a turbulent flow of the liquid around the bubbles and slugs. They disperse into small bubbles forming the churn flows. The spatial and temporal phase distribution inside the minichannel can be used for identification of different two-phase flow patterns in the minichannel.

Due to the high complexity of these phenomena non-periodic (chaotic) flows usually occur. As these flows are difficult to control, possible applications of the microchannel flow systems are significantly reduced. To identify such flows, one has to apply the non-linear analysis, which enables identification of flow patterns. Wang et al. [5] showed that the non-linear analysis allows us to identify flow patterns in a oil-gas-water mixture. The results of a non-linear analysis of temperature and pressure fluctuations in microchannels are discussed by Mosdorf et al. [6]. On the other hand, Wang et al. [7] used the non-linear analysis of the pressure fluctuations to identify the flow patterns of air in water. It has been shown that the methods characterizing the non-linear dynamics of the flow of oil-water [8] are useful for identification of the flow patterns and for assessment of the complexity of these patterns. In addition, Jin et al. [9] showed that the correlation dimension and Kolmogorov entropy are sufficient to identify the flow patterns.

Recently, Faszczewski et al. [10] used the recurrence plot method to analyze the flow patterns in a vertical minichannel, finding that this method enables determination of the parameters which define the borders between flow patterns. Partitioning of air slugs into air bubbles of different sizes and small air bubbles aggregation into larger air bubbles were identified by recurrences $[11,12]$.

Zhou et al. [13] examined the separation contours between two phases during the air-water mixture flow in a horizontal tube by using the fractal dimension, Shannon entropy, the maximal Lyapunov exponent. Finally, the Recurrence Quantification Analysis (RQA) with the characteristic parameters for dynamics identification was also proposed $[14,15]$. In the present paper, we discuss the transition from non-periodic to periodic flow patterns in a horizontal minichannel by using RQA.

\footnotetext{
${ }^{a}$ e-mail: g.litak@pollub.pl (Corresponding author)
} 

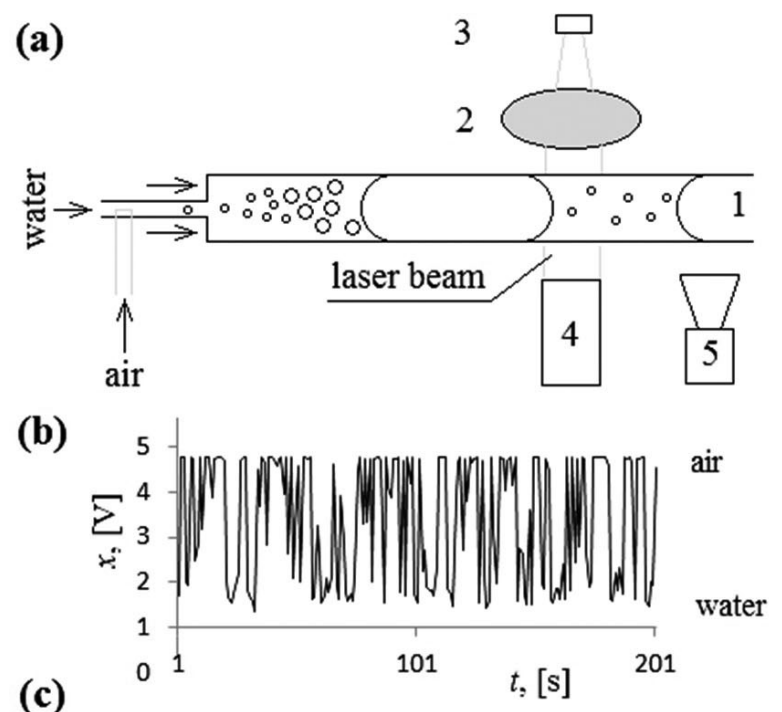

(c)

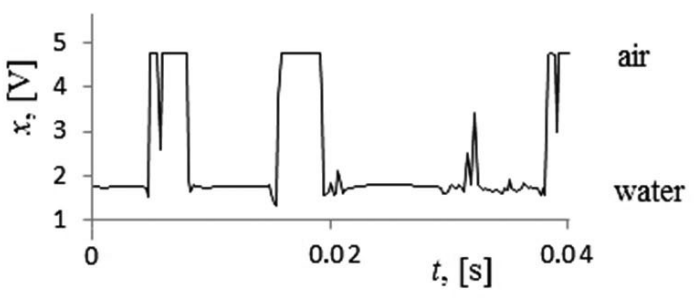

(d)

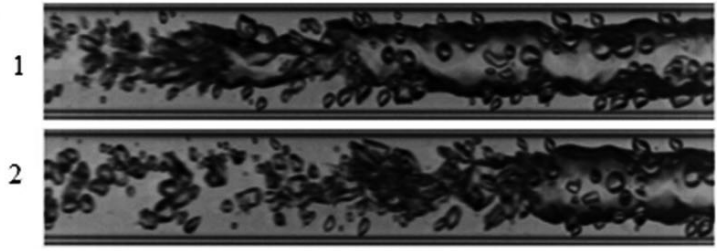

Fig. 1. Schematic design of the experimental stand and examples of recorded data. (a) Experimental setup: 1. horizontally oriented minichannel with a diameter of $2 \mathrm{~mm}, 2$. lens, 3. phototransistor sensor, 4. laser, 5. High speed camera. (b) Signal from laser-phototransistor sensor in the bubble flow. (c) Signal from laser-phototransistor sensor in the slug flow. (d) Two subsequent frames of high-speed video frames showing the slug defragmentation by turbulent liquid flow.

\section{Experimental setup and the measurment procedure}

A schematic design of the experimental stand is shown in fig. 1(a). Flow patterns were recorded with using a Phantom digital camera at $5000 \mathrm{fps}(1280 \times 64$ pixels $)$. The video records were used to identify the mechanism of small bubble creation by a turbulent flow (fig. $1(\mathrm{~d})$ ). The content of the minichannel (i.e. bubbles or liquid) was qualitatively assessed using the laser-phototransistor sensor. A schematic view of the laser-phototransistor sensor is shown in fig. 1(a). The sensor consisted with a laser generating a $3 \mathrm{~mm}$ laser beam, a lens and a silicon sensor placed in the focal point of the lens. Data from the laser-phototransistor sensors were acquired by the acquisition system (an accuracy of $1 \mathrm{mV}$ for voltages in the range of $-10 \mathrm{~V}$ to $10 \mathrm{~V}$ ) at a sampling rate of $5 \mathrm{kHz}$. Examples of the data recorded by the laserphototransistor sensor are presented in figs. 1(b),(c). In the first case (fig. 1(b)), the velocity of the bubble front was $3.37 \mathrm{~m} / \mathrm{s}$ while in the second case (fig. 1(c)) it was $1.85 \mathrm{~m} / \mathrm{s}$. Figure 1(d) illustrates the defragmentation of the air slug caused by vortices. These vortices occur at the end of the slug flow due to the differences in air slugs and water velocities. Here the decreasing width of the slug tail leads to the variations in pressure and the tearing of air bubbles.

Figure 2 shows the digital camera snapshots of the selected flow cases with decreasing the water volume flow rate $q$ from the bottom to top cases. Interestingly, the initial flow pattern (at the bottom of fig. 2) with different sizes of bubbles transformed into a slug flow through a churn phase. Actually, the flow seems to be more regular or/and periodic after the formation of the optimal length slugs. This is the principal effect studied in this paper. For the identification of this transition we propose to use the Recurrence Plots (RP) and Recurrence Quantification Analysis (RQA).

\section{Recurrence analysis}

Generally, the periodicity of the underlying dynamics can be investigated by means of recurrences which is calculated for each point of the reconstructed trajectory. This method was developed by Eckmann et al. [16] and extended by 


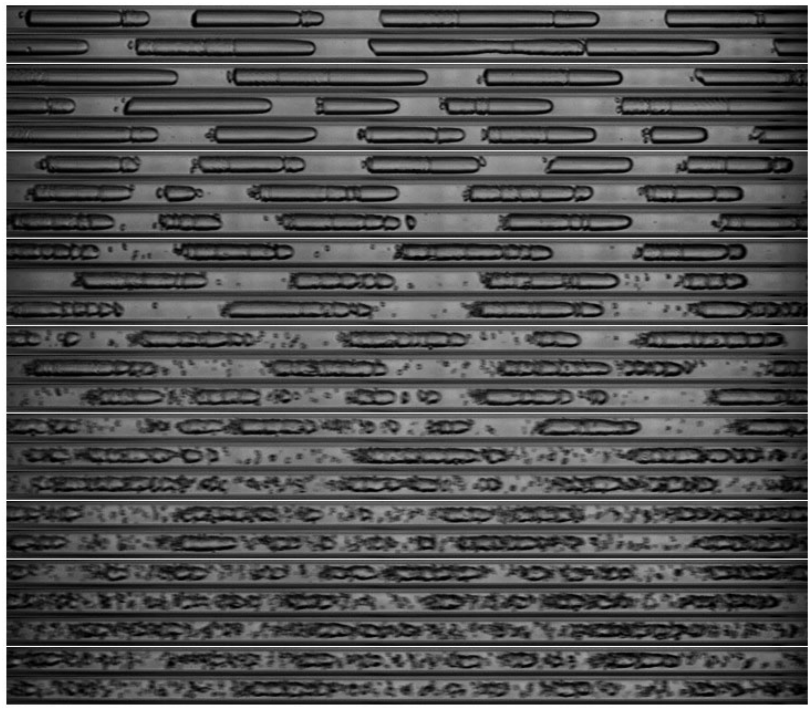

Fig. 2. Photos of the selected flow cases with decreasing water volume flow rate $q[1 / \mathrm{min}]$ ranging from 0.73 at the bottom to 0.11 on the top. The experimental data were collected by a quasistatic change of $q$ from higher to lower values. The corresponding air volume flow rate was $q_{a}=0.1 \mathrm{l} / \mathrm{min}$.
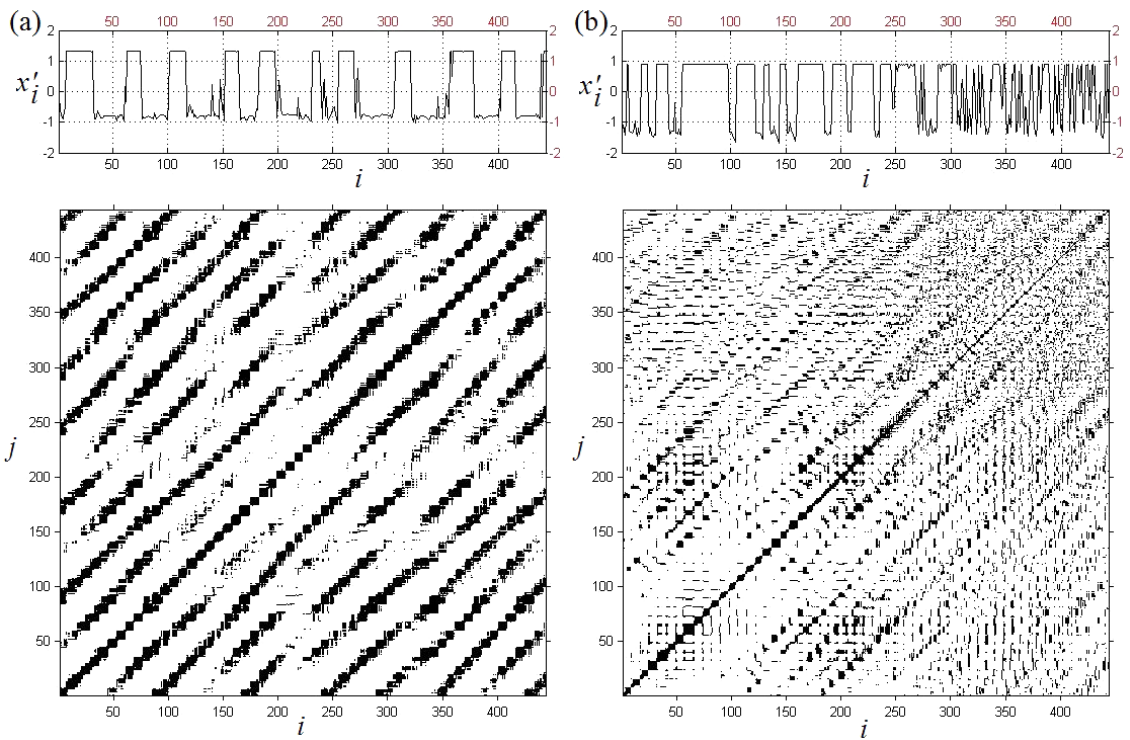

Fig. 3. Laser light transmission and the corresponding recurrence plots of the lines from fig. 2: No. 4 (a) from the top $(q=0.169 \mathrm{l} / \mathrm{min})$ No. 3 (b) from the bottom $(q=0.632 \mathrm{l} / \mathrm{min}) . i, j$ are related to the measurement instants expressed in the sampling units $\Delta t=0.2 \mathrm{~ms}$. For recurrence plots estimations we used the Cross Recurrence Plot Toolbox [24].

Webber and Zbilut [17], Casdagli [18] and later by Marwan and others [19]. This method can be applied for both short deterministic and noisy experimental data (see $[20,21]$ ). Two points on a trajectory are marked as neighbours if they are close enough to each other and this can be expressed by the distance matrix $R$ with its element $R_{i j}^{\epsilon}$ given by [16]:

$$
R_{i j}^{\epsilon}=\Theta\left(\epsilon-\left\|\mathbf{x}_{i}-\mathbf{x}_{j}\right\|\right),
$$

where $\epsilon$ is the threshold value and $\Theta(\cdot)$ denotes the Heaviside function. The number of recurrence points depends on both the underlying dynamics and the threshold value. A standard technique for approximations is that the threshold value should not be higher than several percentage of the total number of points [19].

Figure 3 shows the laser light transmission time series and the corresponding RPs for two different water volume flow rates $q$. In these cases, the RP $\epsilon=3 \sigma$ calculated with Euclidean distance in the reconstructed phase space. The embedding dimension $m=8$, the delay in the reconstructed coordinates $\delta i=8$ calculated by standard methods using the first minimum of averaged mutual information and the nodal fraction of the false neighbours [22]. Following 

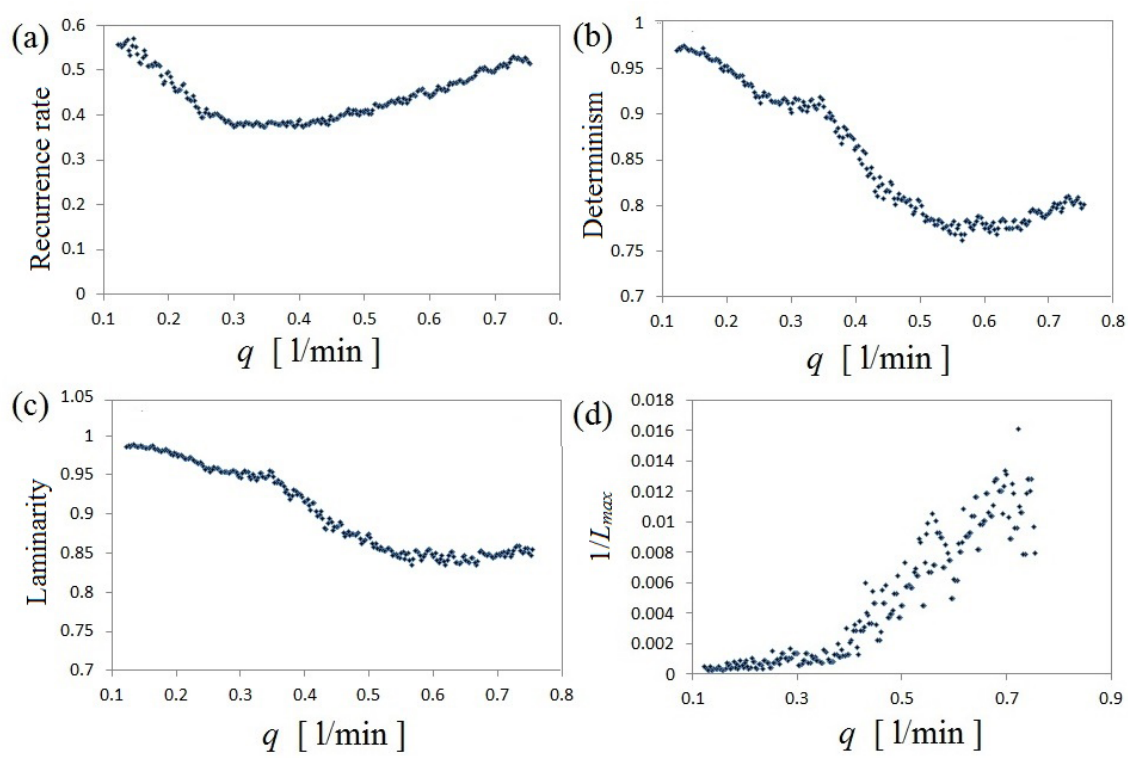

Fig. 4. The RQA results: Recurrence rate - $R R$, Determinism - $D E T$, Laminarity - $L A M$, and $1 / L_{\max }$ as functions of the water volume flow rate $q$ for (a)-(d), respectively. The RQA and embedding assumptions [24] were $\epsilon=3 \sigma$, the embedding parameters as in fig. 3.

Takens [23] we define the following vectors $\mathbf{x}$ based on the laser light transmission $x(t)$ which can be written in the embedded space:

$$
\mathbf{x}(t)=(x(t), x(t-\delta i \Delta t), x(t-2 \delta i \Delta t), \ldots, x(t-(m-1) \delta i \Delta t)),
$$

where $\Delta t$ is a sampling period. $\mathbf{x}_{i}$ and $\mathbf{x}_{j}$ appearing in eq. (1) are related to discrete time $t_{i}=i \Delta t$ and $t_{j}=j \Delta t$, respectively via $\mathbf{x}_{i}$ or $j=\mathbf{x}\left(t_{i}\right.$ or $\left.j\right)$. The horizontal and vertical axes represent the time instants $i$ and $j$ for which the distance formula (eq. (1)) is applied.

Note that for $q=0.169 \mathrm{l} / \mathrm{min}$ (a) the RP clearly indicates the periodic changes signalled by the diagonal lines formed by the recurrence points. On the other hand, the second case for $q=0.632 \mathrm{l} / \mathrm{min}$ (b) shows a definitely nonperiodic behaviour.

To throw more light on the transition we use the RQA parameters [19]. The recurrence rate, $R R$, is a measure the systems ability to return to the neighbourhood of its previous state and has the following definition:

$$
R R=\frac{1}{N^{2}} \sum_{i, j \neq i}^{N} R_{i j}^{\epsilon},
$$

where $N$ is a number of sampling points.

Regarding other parameters the RQA provides the probability $p(l)$ or $p(v)$ of line distribution according to their lengths $l$ or $v$ (for diagonal and vertical lines). They are calculated as

$$
p(z)=\frac{P^{\epsilon}(z)}{\sum_{x=x_{\text {min }}}^{N} P^{\epsilon}(z)},
$$

where $z=l$ or $v$ depend on the diagonal or vertical structures in a specific recurrence plot. $P^{\epsilon}(z)$ denotes the histogram of $z$ lengths and a fixed value of $\epsilon$.

Such measures as determinism $D E T$, laminarity $L A M$, are based on probabilities $P^{\epsilon}(z)$

$$
\begin{gathered}
D E T=\frac{\sum_{l=l_{\text {min }}}^{N} l P^{\epsilon}(l)}{\sum_{l=1}^{N} l P^{\epsilon}(l)}, \\
L A M=\frac{\sum_{v=v_{\min }}^{N} v P^{\epsilon}(v)}{\sum_{v=1}^{N} v P^{\epsilon}(v)},
\end{gathered}
$$

where $l_{\min }$ and $v_{\min }$ denote minimal values which should be chosen for a specific dynamical system. In our case we assumed $l_{\text {min }}=v_{\text {min }}=2$. The determinism $D E T$ is a measure of the predictability of the examined dynamical system 
and provides the ratio of the recurrence points formed in diagonals to all recurrent points. Note that in a periodic system all recurrence points would be included in the lines parallel to the main diagonal. On the other hand, the laminarity $L A M$ is a similar measure which corresponds to the points formed in vertical lines. This measure indicates the dynamics of potential periodic fluctuations. High values correspond to the slow predictable changes, while small ones to sudden changes in dynamics. Finally $L_{\max }$ is the maximum diagonal length (beyond the central diagonal), the inverse of which, $1 / L_{\max }$, can be a measure of non-periodicity (chaoticity) of the examined motion [25].

Figure 4 shows the results of the RQA. Note that the recurrence rate, $R R$, shows the minimum for a moderate water volume flow rate, $q$. This minimum corresponds to the bifurcation region. In the limits of small and large $q$ we observe the slug and bubble flow patterns, while in the middle they are mixed. Both laminarity, DET, and lamininarity, LAM, decrease with increasing $q$, which can be explained by the fact that air bubble velocities depend on their volume and they are stabilized for small $q$ leading to the periodic mass flow transport. This trend is simultaneously confirmed by $1 / L_{\max }$ (fig. $4(\mathrm{~d})$ ) which increases with $q$. Obviously, it signals the occurrence of a chaotic flow of the air-water mixture.

\section{Conclusions}

In summary, it is worth drawing attention to the fact that the minichannel two phase flow is very sensitive to the water volume rate, $q$. The destruction and fragmentation of slugs by churn with increasing $q$, causes the occurrence of various size air bubbles embedding in the water environment. Furthermore, the different size bubbles have different velocities and, thus, introduce various time scales to the dynamical system. These multiple velocities are the main reason for the global change in the flow patterns from the periodic to chaotic flow.

This research was funded by the Polish National Centre of Science under agreement no. DEC-2013/09/B/ST8/02850.

Open Access This is an open access article distributed under the terms of the Creative Commons Attribution License (http://creativecommons.org/licenses/by/4.0), which permits unrestricted use, distribution, and reproduction in any medium, provided the original work is properly cited.

\section{References}

1. L. Zhao, K.S. Rezkallah, Int. J. Multiphase Flow 19, 751 (1993).

2. S. Wongwises, M. Pipathattakul, Exp. Therm. Fluid Sci. 30, 345 (2006).

3. L. Chen, Y.S. Tian, T.G. Karayiannis, Int. J. Heat Mass Transf. 49, 4220 (2006).

4. G.R. Anjos, N. Borhani, N. Mangiavacchi, J.R. Thome, J. Comput. Phys. 270, 366 (2014).

5. Z.Y. Wang, N.D. Jin, Z.K. Gao, Y.B. Zong, T. Wang, Chem. Eng. Sci. 65, 5226 (2010).

6. R. Mosdorf, P. Cheng, H.Y. Wu, M. Shoji, Int. J. Heat Mass Transf. 48, 4667 (2005).

7. S.F. Wang, R. Mosdorf, M. Shoji, Int. J. Heat Mass Transf. 46, 1519 (2013).

8. Y.-B. Zong, N.-D. Jin, Z.-Y. Wang, Z.-K. Gao, C. Wang, Int. J. Multiphase Flow 36, 166 (2010).

9. N.D. Jin, X.B. Nie, Y.Y. Ren, X.B. Liu, Flow Meas. Instrum. 14, 169 (2003).

10. M. Faszczewski, G. Górski, R. Mosdorf, Acta Mech. Autom. 6, 31 (2012).

11. G. Górski, G. Litak, R. Mosdorf, A. Rysak, Eur. Phys. J. B 88, 239 (2015).

12. G. Górski, G. Litak, R. Mosdorf, A. Rysak, Z. Naturforsch. A 70, 843 (2015).

13. Y. Zhou, H. Li, Y. Miao, Flow Meas. Instrum. 30, 18 (2013).

14. S. Sultornsanee, I. Zeid, S. Kamarthi, Proc. Comp. Sci. 6, 375 (2011).

15. S. Sultornsanee, Interdisciplinary Engineering Dissertations, http://hdl.handle.net/2047/d20002559 (2012).

16. J.-P. Eckmann, S.O. Kamphorst, D. Ruelle, Europhys. Lett. 5, 973 (1987).

17. C.L. Webber Jr., J.P. Zbilut, Appl. Physiol. 76, 965 (1994).

18. M.C. Casdagli, Physica D 108, 12 (1997).

19. N. Marwan, M.C. Romano, M. Thiel, J. Kurths, Phys. Rep. 438, 237 (2007).

20. G. Litak, A. Syta, J. Gajewski, J. Jonak, Meccanica 45, 603 (2010).

21. G. Litak, A. Syta, R. Rusinek, Int. J. Adv. Manufact. Technol. 56, 445 (2011).

22. H. Kantz, T. Schreiber, Non-linear Time Series Analysis (Cambridge University Press, Cambridge, 1997).

23. F. Takens, Detecting Strange Attractors in Turbulence, Dynamical Systems and Turbulence, edited by D.A. Rand, L.-S. Young, in Lecture Notes Math., Vol. 898 (Springer, Berlin, 1981) pp. 366-381.

24. N. Marwan, Cross Recurrence Plot Toolbox for Matlab, Ver. 5.15, Release 28.10, http://tocsy.pik-potsdam.de (2011).

25. N. Marwan, J. Kurths, S. Foerster, Phys. Lett. A 379, 894 (2015). 